\title{
Shortness of filum terminale represents an anatomical specific feature in fibromyalgia: a nuclear magnetic resonance and clinical study
}

\author{
Roberto Mantia ${ }^{1}$ \\ Marco Di Gesù 1 \\ Angelo Vetro ${ }^{1}$ \\ Fabrizio Mantia ${ }^{1}$ \\ Sebastiano Palma \\ Angelo lovane $^{3}$ \\ 1 Medical Center Mantia, Palermo, Italy \\ 2 Radiology Institute, University of Palermo, Italy \\ 3 Sport and Exercise Sciences Research Unit, Uni- \\ versity of Palermo, Italy
}

Corresponding author:

Marco Di Gesù

Medical Center Mantia

Via Francesco Ferrara

90141 Palermo, Italy

E-mail: m.digesu@centromedicomantia.it

\section{Summary}

Background: we aimed to assess whether shortness of filum terminale (FT) can represent a specific feature of fibromyalgia. Therefore we investigated benefits coming from FT section with a mini-invasive technique in patients with fibromyalgia. Filum terminale disease (FD), described firstly in 1996 , is consequence of an abnormal traction exerted on spinal cord since FT is shorter than usual. Fibromyalgia syndrome (FS) is featured by chronic widespread musculoskeletal pain associated with stiffness and extra-skeletal symptoms affecting many organs and systems. Filum terminale disease and fibromyalgia syndrome share common clinical features in at least one subset of patients. Methods: we evaluated 42 patients firstly diagnosed for FS and then re-evaluated by nuclear magnetic resonance. 38 out of $\mathbf{4 2}$ had also FD and 20 of them underwent surgical treatment, i.e., FT section according to Royo-Salvador technique.

Results: after physical therapy, surgically treated patients showed significant improvement of symptoms in terms of reduction of pain and increment of quality of life, compared to group, which refused surgery and performed physical therapy only.

Conclusion: we suggest that FT shortness can be considered one of predisposing causes for developing FS and that FD surgical treatment in patients with FS can improve overall treatment outcome.
KEY WORDS: anatomical modification, fibromyalgia impact questionnaire, fibromyalgia assessment status, mini-invasive surgery, musculo-skeletal disorder, physical therapy.

\section{Introduction}

Filum terminale (FT) is a strand of fibrous tissue about $20 \mathrm{~cm}$ long and made of two parts: filum terminale internum and filum terminale externum. The former is nearly entirely made of pia mater, the innermost meningeal layer, and extends from the conus medullaris of the spinal cord to the cul-de-sac of the most external meningeal layers, i.e., the arachnoid membrane and the dura mater. At this level, it fuses with those meningeal membranes thus making the coccygeal ligament, which fastens the most distal portion of the medulla to the coccyx. Indeed, the coccygeal ligament is often referred as filum terminale externum.

Some diseases affecting FT have been described, including some forms of tumors, mineral deposition and filum terminale disease (FD), described in 1996 by Royo-Salvador ${ }^{1,2}$. Filum terminale disease is an outcome of the abnormal traction exerted on the spinal cord by a FT shorter that usual. This condition is commonly diagnosed by means of nuclear magnetic resonance since the conus medullaris results to be more distal than normally in adult individuals, i.e., behind the body of a vertebra comprised between the twelfth thoracic (T12) and the first lumbar (L1) vertebrae. Most importantly, within a physiological variability, nuclear magnetic resonance scans show more distal cerebellar tonsils as well, being behind the greater occipital foramen. Clinical symptoms concern the central nervous system (CNS), mainly due to the traction of spinal cord and brain stem. Secondary symptoms are commonly present at ocular, oropharyngeal, circulatory, urinary, digestive and endocrine levels ${ }^{3}$. Filum terminale disease is a syndrome featured by a slow progression. At the early stage, the patient is usually not experiencing any symptoms. On the basis of anatomo-clinical data, section of FT has been proposed as a surgical mini-invasive approach able to stop the progression of the symptoms caused by the spinal traction ${ }^{4}$.

Fibromyalgia, or fibromyalgia syndrome (FS), is a disease mostly represented in the female gender (female/male ratio: 6/1). Overall incidence is between one and eight percent ${ }^{5,6}$. It can affect at any age and its incidence increases with age ${ }^{7}$. Fibromyalgia syndrome is defined primary, or idiopathic, when not 
combined with any other pathology, while it is defined secondary when it is diagnosed in association with other clinical conditions (mostly chronic diseases) ${ }^{5,6}$. In any case, FS is featured by chronic widespread musculoskeletal pain associated with stiffness and extra-skeletal symptoms affecting several organs and systems ${ }^{5}$. Among these one, very frequent are bowel pain, headache, tachycardia, menstrual disorders, breath shortness, anxiety and low mood and depression. It is also featured by intense tiredness and easy fatigability ${ }^{7}$. Functional imaging studies support the theory that many signs and symptoms of FS may be due to a CNS dysfunction and, a consequently altered processing of sensory stimuli8-10. Findings of hypo-perfusion in thalamus ${ }^{11}$ and caudate nucleus ${ }^{12}$, by single photon emission computed tomography scan analysis of patients with FS, support this hypothesis.

The present study aimed to evaluate whether shortness of FT can represent a specific feature of fibromyalgia. In particular, we compared overall outcome of a group of subjects affected by FS, which underwent firstly surgery for section of FT and then physical therapy, to outcome of a group of patients, which refused surgery and performed physical therapy only.

\section{Materials and methods}

From March 2009 until December 2012, we followed 42 (38 women and 4 men) patients with diagnosis of FS. Since drug treatment (analgesics, antidepressants, anti-seizures, non-steroid anti-inflammatories) gave unsatisfactory results or only transient benefits, they underwent our screening for physical therapy candidates. The exclusion of criteria was the presence of other rheumatologic, neurological and immunological diseases. Hence, all patients were clinically reevaluated, in blind, by three expert neuro-physiatrists (MDG, AV, RM). In terms of radiological evaluation, the patients were examined by means of nuclear magnetic resonance of the cervical and lumbar spine. In some cases, it was not the position of the medullary cone (between the first lumbar vertebrae and the second or third one) but the displacement of the cerebellar tonsils (behind the greater occipital foramen) to allow assessing the diagnosis of FD. Therefore patients were re-assessed by means of two clinical scales for quality of life, i.e., the Fibromyalgia Impact Questionnaire (FIQ) and the self-administered Fibromyalgia Assessment Status (FAS) ${ }^{13,14}$.

In 38 over 42 patients ( 36 women and 2 men), we diagnosed a FD and all these 38 patients were enrolled in our study. All patients were divided in surgery+ physical therapy group (SPT) and physical therapy group (PT) according to their free consent to surgery. Surgery+physical therapy group ( $n=20$, all women, age $51 \pm 6$ yrs, body mass $61 \pm 5 \mathrm{~kg}$, body height $165 \pm 6$ $\mathrm{cm}$ ) decided to undergo surgical section of FT (specifically, filum terminale externum) and subsequently rehabilitation protocol. Surgical intervention was per- formed in "Hospital Sanitas Cima" in Barcelona, Spain. Physical therapy group ( $n=18,16$ women and 2 men, age $52 \pm 5.1 \mathrm{yrs}$, body mass $68 \pm 12 \mathrm{~kg}$, body height $168 \pm 9 \mathrm{~cm}$ ) refused surgical treatment and therefore underwent only the rehabilitation protocol. The study conformed to the Declaration of Helsinki 1964 and was conducted after approval from the local Medical Center Ethics Committee, which followed the standard of the Muscles, Ligaments and Tendons Journal ${ }^{15}$. The procedures, risks and goals were explained to the participants and written consent was obtained prior to participation.

\section{Experimental setting}

After surgery nuclear magnetic resonance of cervical and lumbar spine was repeated in SPT to assess morphological changes, if present. Both groups were evaluated in two different days in laboratory in Centro Medico Mantia (Palermo, Italy), in temperature/humidity comfortable conditions by means of FIQ and FAS scales. To balance for the time effect, SPT was clinically evaluated before section of FT (T0) and after four-week recovery+nine-week physical therapy (T1). Correspondingly, ST was assessed before study start (TO) and after four-week rest+nine-week physical therapy (T1).

\section{Physical therapy}

Physical therapy was standardized and provided to all patients. All exercise sessions were administered by accredited and experienced physiotherapists graduated in Exercise Science, as well. Program included 27 sessions (three per week, for nine weeks), 60 minutes each, with postural exercises for the back associated with active aerobic exercises and treatment of painful areas with transcutaneous electrical nerve stimulation (Gymna Combi 200, GymnaUniphy, Bilzen, Belgium). Routines were designed to keep a low intensity and basically included static and dynamic exercises for posture and balance (performed in presence and absence of visual input) as well as joint and spine mobility, according to a previous study ${ }^{16}$. Patient did not take painkiller medication for all the rehabilitation period, except for free assumption of acetaminophen $(1000 \mathrm{mg})$ in case of need.

\section{Statistical analysis}

Descriptive statistics are presented as mean $( \pm S D)$. Student's $t$-test for independent samples was used to detect for any initial differences between groups for anthropometric measures. Starting from statistical power of ANOVA, we calculated total sample size with G-Power 3.1.3. When considering main factors of present study, a sample size of 8 for each group showed to be appropriate for a statistical power greater than 0.9. To assess reliability and variability of the measures, we 
Shortness of filum terminale represents an anatomical specific feature in fibromyalgia: a nuclear magnetic resonance and clinical study

calculated coefficient of variation ( $C V=S D / m e a n \%)$. The FIQ and FAS scores were analysed by using a separated two-way ANOVA with repeated measures and intervention type as factor and Bonferroni post-hoc test. Assumption of normality was verified by using Shapiro-Wilk Test. "Between" factor was treatment kind with two levels (SPT and PT) and "within" factor was time with two levels (pre- and post-treatment). Etasquared was calculated to assess effect size. Spearman's correlation coefficients were computed for FIQ and FAS within the two groups. Pair-wise comparison was performed when the main effect was significant and significance level was set at $p<0.05$. Statistical analysis was performed by using SigmaPlot 11.0 software (Systat Software, Tulsa, OK, USA).

\section{Results}

All data are reported in Table 1. There was no difference ( $p>0.05)$ between groups at baseline conditions for age and body height, while there was significantly difference $(p=0.03)$ for body mass. Comparison between the nuclear magnetic resonance images of SPT obtained before and after the section of FT did not show any significant morphological change (Fig. 1). After one year of the treatment, all patients showed both displacements of cerebellar tonsil behind greater occipital foramen and medullar cone ending at second or third lumbar vertebra level either before or after surgery. Although there were no evident anatomical modifications at nuclear magnetic resonance imaging level, patients experienced a significant amelioration of symptoms.

In spite of the FIQ and FAS scores variability at baseline (CV up to 15\%), the statistical power remained high in relation to sample power $(>8)$. The analysis of variance revealed for $F I Q$ a treatment effect $\left(F_{(1,37)}=120.790, p<0.0001, \eta^{2}=0.770\right)$ and a treatment type effect $\left(F_{(1,37)}=108.117, p<0.0001, \eta^{2}=0.750\right)$. Posthoc analysis showed significant FIQ decreases of 70 and $13 \%(p<0.0001$ and $p<0.001)$ after SPT and PT, respectively (Fig. 2). Similarly, ANOVA revealed for FAS a treatment effect $\left(F_{(1,37)}=114.459, p<0.0001\right.$,

Table 1. Absolute and percent changes for each variable after treatments.

\begin{tabular}{lllllll}
\hline & Pre & Post & $(\Delta \%)$ & Pre & Post & $(\Delta \%)$ \\
\hline Group & & FIQ & & & FAS & \\
\hline SPT & $69.1 \pm 13.2$ & $20.8 \pm 7.5$ & $-70^{*}$ & $6.2 \pm 0.9$ & $1.4 \pm 1.0$ & $-77^{*}$ \\
PT & $82.0 \pm 6.2$ & $71.6 \pm 13.1$ & $-13^{*}$ & $7.2 \pm 0.8$ & $6.1 \pm 1.0$ & $-15^{*}$ \\
\hline
\end{tabular}

Values are expressed as mean \pm SD Fibromyalgia Impact Questionnaire (FIQ) and self-administered Fibromyalgia Assessment Status (FAS) scores for surgery+physical therapy group (SPT) and physical therapy group (PT); ${ }^{*} p<0.001$ versus baseline conditions.

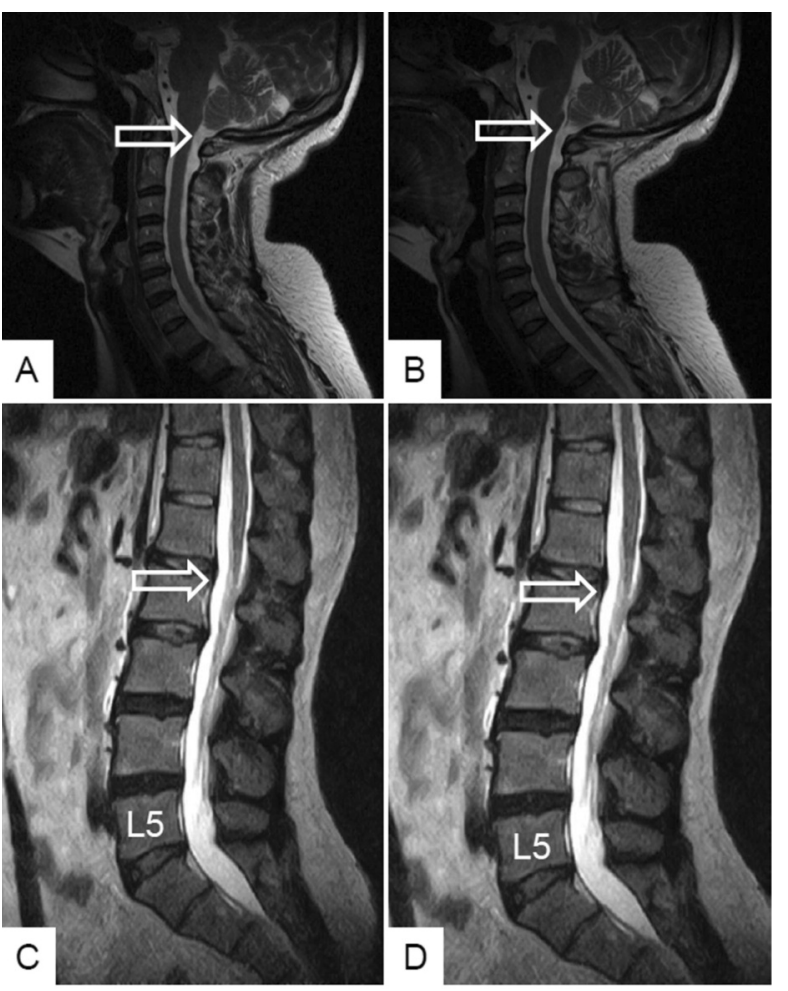

Figure 1 A-D. Relevant cervical (A-B) and lumbar (C-D) spine nuclear magnetic resonance images in patients with Fibromyalgia syndrome and Filum terminale disease. Images have been taken in a patient before $(A, C)$ and after $(B, D)$ section of FT. In particular, images show the distal position of cerebellar tonsils (arrow) before $(A)$ and after (B) surgery and medullary cone (arrow) ending at second lumbar vertebra level both before (C) and after (D) surgery. $\mathrm{L} 5=$ fifth lumbar vertebra. 


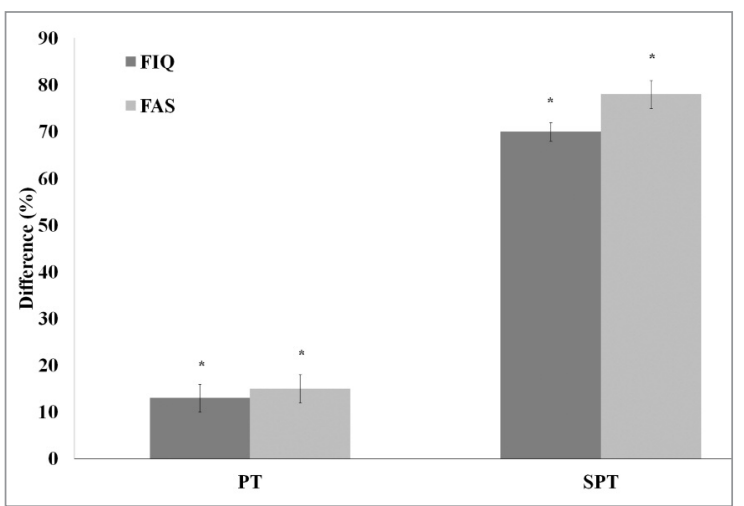

Figure 2. Fibromyalgia Impact Questionnaire (FIQ) and self-administered Fibromyalgia Assessment Status (FAS) change $(\Delta \%)$ between pre- and post-treatment in physical therapy group (PT) and surgery+physical therapy group (SPT). Data are showed as mean \pm SE, "*” represent significantly effect with $\mathrm{p}<0.001$.

$\left.\eta^{2}=0.761\right)$ and a treatment type effect $\left(F_{(1,37)}=145.369\right.$, $\left.\mathrm{p}<0.0001, \eta^{2}=0.802\right)$. Post-hoc analysis showed significant FAS decreases of 75 and $15 \%(p<0.0001$ and $\mathrm{p}<0.0001$ ) after SPT and PT, respectively (Fig. 2). Significant correlation was found between FIQ and FAS in SPT both before $(r=0.467, \mathrm{p}=0.038)$ and after treatment $(r=0.782, \mathrm{p}<0.0001)$. Differently, in PT negative not significant correlation was found before treatment ( $r=-$ $0.400, p>0.100)$ and significant correlation was found after that $(r=0.477, \mathrm{p}=0.045)$.

\section{Discussion}

Main results of this study come from comparison between pre- and post-treatment scores in the two groups. Patients treated with surgical section of filum terminale (FT) and subsequent rehabilitation treatment showed greater improvements in terms of reduced pain and improved quality of life compared to patients treated exclusively with rehabilitation. Further clinical follow-up may assess results after longer periods since end of treatment to evaluate retention of obtained benefits.

To the best of our knowledge, there are no data in literature rebutting the hypothesis that fibromyalgia syndrome (FS) may be due to FT shortness. By contrast, there is a number of reports supporting strong correlation between FS and central nervous system diseases. In particular, there are many evidences about neuroendocrine and autonomic impairment in FS with symptoms very similar to those occurring in a high percentage of filum terminale disease (FD) patients ${ }^{17-19}$. These prompted us to state that, at least in a limited subset of patients, FS may be considered an epiphenomenon of FD. Practical relevance of such reasoning lies in the suggestion that patients should be treated for FD, i.e., by FT section before performing physical therapy to improve their outcome.
Royo-Salvador 1,2,4,20-22 developed the theory that Arnold-Chiari syndrome, syringomyelia and idiopathic scoliosis have a common etiology: medullary traction due to shortness of FT. Garceau ${ }^{23}$ previously described symptoms related to spinal cord traction and reported the cases of three patients with paraparesis, scoliosis, headache and dysesthesia, which recovered after section of FT. Roth ${ }^{24}$ hypothesized that stretching of spinal cord is responsible for scoliosis and syringomyelia, and described a case of shortness of $\mathrm{FT}$ with subsequent development of syringomyelia, which resolved after sectioning of $\mathrm{FT}^{25}$.

Nevertheless, our data do not show any anatomical change in terms of displacement of cerebellar tonsil and of medullary cone in our patients after surgery. This is probably due to the fact that the patients, who underwent our treatment, had suffered from this condition since many years before its diagnosis. Therefore, connective tissues joining dura mater to surrounding periosteum could have established a particularly strong binding between these structures. Further follow-up could include further nuclear magnetic resonance examination, since it is possible that, after some years since surgery, we could find some visible changes in the positions of both cerebellar tonsil and medullary cone. We acknowledge to have investigated two "locked" intervention modalities: surgery AND physical therapy and physical therapy ONLY. In so doing, it remains clearly unknown how much of the benefit for the patients who underwent surgery AND physical therapy were due to surgery OR physical therapy. Further investigations will need to address this issue.

In conclusion, our data allow stating that a shortness of FT, such as the one featuring FD, may be responsible of FS. We suggest evaluating, by nuclear magnetic resonance, anatomical position the cerebellar tonsil and medullary cone in all patients diagnosed with FS. Treatment of FD is a minimally invasive and little expensive surgical technique. Therefore it is likely that in the forthcoming years this technique will become available in a number of clinical centers and used as new therapeutic tool for treatment of FS, currently considered an incurable disease.

\section{Acknowledgements}

The authors gratefully thank all patients who took part in the study.

\section{Competing interest}

The authors declare non conflict of interest relate to the present article.

\section{Funding}

No funding was received for this investigation. 
Shortness of filum terminale represents an anatomical specific feature in fibromyalgia: a nuclear magnetic resonance and clinical study

\section{Author contribution}

$\mathrm{RM}, \mathrm{MDG}, \mathrm{AV}, \mathrm{FM}$ and $\mathrm{Al}$ were involved in the design of the experiment, collection of the data, data analysis and interpretation and writing of the manuscript. $\mathrm{SP}, \mathrm{FM}$ and MDG were involved in the data acquisition and data analysis (especially the statistical analyses), and writing of the manuscript.

\section{References}

1. Royo-Salvador MB. Syringomyelia, scoliosis and idiopathic Arnold-Chiari malformations: a common etiology. Rev Neurol. 1996a;24:937-959.

2. Royo-Salvador MB. Platybasia, basilar groove, odontoid process and kinking of the brainstem: a common etiology with idiopathic syringomyelia, scoliosis and Chiari malformations. Rev Neurol. 1996b;24:1241-1250.

3. Yamada S, Zinke DE, Sanders D. Pathophysiology of "tethered cord syndrome". J Neurosurg. 1981;54:494-503.

4. Royo-Salvador MB. A new surgical treatment for syringomyelia, scoliosis, Arnold-Chiari malformation, kinking of the brainstem, odontoid recess, idiopathic basilar impression and platybasia. Rev Neurol. 1997;140:523-530.

5. Cazzola M, Sarzi Puttini P, Stisi S, et al. Fibromyalgia syndrome: definition and diagnostic aspects. Reumatismo. 2008;60:3-14.

6. Claw DJ. Fibromyalgia: an overview. Am J Med. 2009;122:S3S13.

7. Forseth KO, Gran JT, Husby G. A population study of the incidence of fibromyalgia among women aged 26-55 yr. Brit J Rheumatol. 1997;36:1318-1323.

8. Demitrack M, Crofford L. Evidence for and pathophyiologic implication of hypothalamic-pituitary-adrenal axis dysregulation in fibromyalgia and chronic fatigue syndrome. Ann N Y Acad Sci. 1998;840:684-697.

9. Winfield JB. Fibromyalgia and related central sensitivity syndromes: twenty-five years of progress. Semin Arthritis Rheum. 2007;36:335-338.

10. Yunus MB. Central Sensitivity syndromes: a new paradigm and group nosology for fibromyalgia and overlappiung conditions, and the related issue of disease versus illness. Semin Arthritis Reum. 2008;37:339-352.
11. Kwiatek R, Barnden L, Tedman R, et al. Regional cerebral blood flow in fibromyalgia: single-photon-emission computed tomography evidence of reduction in the pontine tegmentum and thalami. Arthritis Rheum. 2000;43:2823-2233.

12. Mountz JM, Bradley LA, Modell JG, et al. Fibromyalgia in women. Abnormalities in regional cerebral blood flow in the thalamus and caudate nucleus are associated with low pain thresholds. Arthritis Rheum. 1995;38:926.

13. Burckhardt CS, Clark SR, Bennett RM. The fibromyalgia impact questionnaire: development and validation. J Rheumatol. 1991;18:728-733.

14. Michielsen HJ, De Vries J, Van Heck GL. Psychometric qualities of a brief self-rated fatigue measure: The Fatigue Assessment Scale. J Psychosom Res. 2003;54:345-352.

15. Padulo J, Oliva F, Frizziero A, Maffulli N. Muscles, Ligaments and Tendons Journal. Basic principles and recommendations in clinical and field science research. MLTJ. 2013 4:250-252

16. Pau M, Leban B, Collu G, Migliaccio GM. Effect of light and vigorous physical activity on balance and gait of older adults. Arch Gerontol Geriatr. 2014;S0167-4943(14)00111-3.

17. Crofford LJ. Neuroendocrine abnormalities in fibromyalgia and related disorders. Am J Med Sci. 1998;315:359-366.

18. Tanriverdi F, Karaca Z, Unluhizarci K, Kelestimur F. The hypothalamo-pituitary-adrenal axis in chronic fatigue syndrome and fibromyalgia syndrome. Stress. 2007;10:13-25.

19. Staud R. Autonomic dysfunction in fibromyalgia syndrome: postural orthostatic tachycardia. Curr Rheumatol Rep. 2008;10:463-466.

20. Royo-Salvador MB. "Pseudo"sidiopathic scoliosis in syringomyelia. Eur Spine J. 1999;8:421.

21. Royo-Salvador MB. Relating to classification and etiology of Chiari I malformation. Magn Reson Imaging. 1999;17:1403.

22. Royo-Salvador MB, Solé-Llenas J, Doménech JM, GonzàlezAdrio R. Results of the section of the filum terminale in $20 \mathrm{pa}-$ tients with syringomyelia, scoliosis and Chiari malformation. Acta Neurochir (Wien). 2005;147:515-523.

23. Garceau GJ. The filum terminale syndrome (the cord-traction syndrome). J Bone Joint Surg Am. 1953;35:711-716.

24. Roth M. Idiopathic scoliosis from the point of view of the neuroradiologist. Neuroradiology. 1981;21:133-138.

25. Roth M. Cranio-cervical growth collision: another explanation of the Arnold-Chiari malformation and of basilar impression. Neuroradiology. 1986;28:187-194. 